\title{
Rapamycin Ameliorates Proteinuria and Restores Nephrin and Podocin Expression in Experimental Membranous Nephropathy
}

\author{
Stavros Stratakis, ${ }^{1}$ Kostas Stylianou, ${ }^{1}$ Ioannis Petrakis, ${ }^{1}$ Vasiliki Mavroeidi, ${ }^{1}$ \\ Rafaela Poulidaki, ${ }^{1}$ Christina Petra, ${ }^{1}$ Demitrios Moisiadis, ${ }^{1}$ Spyros Stratigis, ${ }^{1}$ \\ Eleftheria Vardaki, ${ }^{1}$ Lydia Nakopoulou, ${ }^{2}$ and Eugene Daphnis ${ }^{1}$ \\ ${ }^{1}$ Nephrology Department, Heraklion University Hospital, 71110 Heraklion, Greece \\ ${ }^{2}$ Pathology Department, Kapodistrian University of Athens, 11527 Athens, Greece
}

Correspondence should be addressed to Kostas Stylianou; kstylianu@gmail.com

Received 26 March 2013; Revised 28 July 2013; Accepted 1 August 2013

Academic Editor: Xuan Zhang

Copyright (C) 2013 Stavros Stratakis et al. This is an open access article distributed under the Creative Commons Attribution License, which permits unrestricted use, distribution, and reproduction in any medium, provided the original work is properly cited.

Objective. Recent studies have shown a beneficial effect of rapamycin in passive and active Heymann Nephritis (HN). However, the mechanisms underlying this beneficial effect have not been elucidated. Methods. Passive Heymann Nephritis (PHN) was induced by a single intravenous infusion of anti-Fxl in 12 Sprague-Dawley male rats. One week later, six of these rats were commenced on daily treatment with subcutaneous rapamycin $0.5 \mathrm{mgr} / \mathrm{kg}$ (PHN-Rapa). The remaining six rats were used as the proteinuric control group (PHN) while six more rats without PHN were given the rapamycin solvent and served as the healthy control group (HC). All rats were sacrificed at the end of the 7th week. Results. Rapamycin significantly reduced proteinuria during the autologous phase of PHN. Histological lesions were markedly improved by rapamycin. Immunofluorescence revealed attenuated deposits of autologous alloantibodies in treated rats. Untreated rats showed decreased glomerular content of both nephrin and podocin whereas rapamycin restored their expression. Conclusions. Rapamycin monotherapy significantly improves proteinuria and histological lesions in experimental membranous nephropathy. This beneficial effect may be mediated by inhibition of the alloimmune response during the autologous phase of PHN and by restoration of the normal expression of the podocyte proteins nephrin and podocin.

\section{Introduction}

Membranous nephropathy (MN) is a common cause of nephrotic syndrome (NS), accounting for approximately $20 \%$ of cases in Caucasians [1]. MN is characterized by thickening of the glomerular basement membrane (GBM) and deposition of immune complexes and complement on its subepithelial aspect.

Spontaneous complete or partial remission of proteinuria occurs in $5-32 \%$ and $25-40 \%$, respectively, at five years [25]. The probability of end-stage renal disease (ESRD) in untreated patients is approximately $15 \%$ at five years, $35 \%$ at 10 years, and $40 \%$ at 15 years $[2-4,6]$. Due to the relatively benign clinical course, immunosuppressive agents are considered only in patients at risk of progressive disease or with severe symptomatic NS $[2,7,8]$.
Recent evidence suggests that the majority of patients with idiopathic $\mathrm{MN}$ have circulating antibodies against phospholipase A2 receptor (PLA2R), which is present on podocytes, as is megalin in rat models of MN [9]. Similarly, neutral endopeptidase has been found as the target antigen in newborns' podocytes with alloimmune neonatal membranous nephropathy [10] and cationic bovine serum albumin as a planted antigen in early childhood MN [11]. Additional circulating autoantibodies against human podocytic antigens have recently been described [12]. It is speculated that as a result of podocyte injury by complement, various intracellular proteins and cryptic epitopes may be exposed, thus inducing "a second wave of immunisation" $[13,14]$.

Heymann Nephritis is a faithful experimental model of the disease that has been extensively studied since first described by Heymann et al. in 1959 [15]. The active model 
of $\mathrm{HN}$ is induced by immunization of Lewis rats with preparations of brush-border proteins. The passive model of $\mathrm{HN}$ (PHN) is induced by a single i.v. injection of heterologous anti-brush border antiserum (anti-FxlA) that produces heterologous IgG subepithelial deposits within hours to days. Proteinuria occurs in almost all animals within five days. This "heterologous phase" is followed, two weeks later, by an "autologous phase" during which rat IgG antibodies are produced against the heterologous IgG. The autologous IgG alloantibodies are also deposited at the subepithelial space, inducing a further increase in proteinuria [16]. The second (autologous) phase of PHN mimics idiopathic $\mathrm{MN}$ because during the autologous phase there is production of autoantibodies (similar to human disease), against a planted exogenous antigen (similar to cationic bovine serum albumin in humans) but also against neoantigens that are exposed in the subepithelial space during the initial injury (again similar to the second wave of immunization that is believed to happen in human idiopathic MN).

Therefore it is the autologous phase of passive $\mathrm{HN}$ that shares the same pathophysiological mechanisms to those recently identified in idiopathic $\mathrm{MN}$ in humans.

To date the therapeutic approach has not changed substantially. The monthly alteration of cyclophosphamide or cyclosporine and corticosteroids remains the standard therapy for severe and persisting proteinuria. Given the significance of IgG antibodies in MN, strategies to target B lymphocytes and antibody formation may be effective in inducing remission of the NS [14]. Indeed there is recent evidence that anti-CD20 antibody administration can effectively treat patients with idiopathic MN $[17,18]$.

The immunosuppressive effect of rapamycin was first attributed to the inhibition of cytokine-induced proliferation and clonal expansion of $\mathrm{T}$ cells. More recently, it has become evident that rapamycin (in contrast to tacrolimus and cyclosporine) inhibits the proliferation of B cells [19] and restricts B cells capable of producing immunoglobulins [20].

Bonegio et al. demonstrated that low dose rapamycin ameliorated proteinuria in experimental PHN and limited tubulointerstitial inflammation and interstitial fibrosis in association with reduced expression of proinflammatory and profibrotic genes [21]. The beneficial effects of rapamycin have also been observed in active HN [22]. Here we tried to investigate more specific effects of rapamycin, beyond the known antifibrotic ones. In particular we examined the effect of rapamycin on podocytes architecture and slit diaphragm proteins, as well as on the deposition of pathogenic autoantibodies that coincides with the autologous phase of PHN.

\section{Materials and Methods}

2.1. Experimental Design. Eighteen male Sprague-Dawley rats (Pasteur Institute, Athens, Greece) were used in this study. The experiment was carried out in accordance with current legislation on animal experiments in the European Union and approved by our institution's Safety and Ethics Committee for Animal Research. All animals were housed in a room with $12 \mathrm{~h}$ light $/ 12 \mathrm{~h}$ dark cycle, constant temperature of $22^{\circ} \mathrm{C}$, and had free access to standard diet and water. PHN was induced in 12 rats by a single i.v. infusion of $0.5 \mathrm{~mL}$ sheep anti-Fxl per 100 gr of body weight. Anti-Fxl antiserum was kindly provided by Dr. Kerjaschki. Rats were anesthetized by intraperitoneal infusion of Ketamine $67 \mathrm{mg} / \mathrm{kg}$ and Xylazine $10 \mathrm{mg} / \mathrm{kg}$.

One week after anti-Fxl infusion all rats became proteinuric. Six of them were randomly selected to commence daily subcutaneous injections of rapamycin (Sigma, St Louis, MO, USA) at a dose of $0.5 \mathrm{mgr} / \mathrm{kg}$ (PHN-Rapa group). Another six rats with $\mathrm{PHN}$ were given subcutaneously only the rapamycin solvent (DMSO) and served as the passive $\mathrm{HN}$ proteinuric group (PHN group). The remaining six, age and weight matched healthy rats without PHN, received only DMSO and served as the healthy control group (HC group). Urine collections were performed weekly in metabolic cages (Tecniplast, Italy). Body weight was also determined weekly and rapamycin dose was adjusted accordingly. All animals were sacrificed 7 weeks after anti-Fxl administration.

2.2. Isolation of Glomeruli. Glomeruli were isolated by differential sieving by utilizing sieves (Retsch, Haan, Germany) of different pore sizes: $150 \mu \mathrm{m}, 106 \mu \mathrm{m}$, and $75 \mu \mathrm{m}$. Isolated glomeruli were retained on the bottom screen of $75 \mu \mathrm{m}$ pore size. Purity of the glomerular isolate was estimated to be $>95 \%$. After several washings with PBS, glomeruli were collected and centrifuged for $4 \mathrm{~min}$ at 1200 r.p.m. The pellet was homogenized in RIPA-buffer containing protease inhibitors and was stored in $-80^{\circ} \mathrm{C}$ till analysis.

2.3. Western Blot (WB) Analysis. Kidney cortex tissue was homogenized in RIPA-buffer containing protease inhibitors (Roche Diagnostics, Hellas, SA). Forty $\mu$ g of glomerular lysate was electrophorized per lane on 7.5\% SDS-gels. The proteins were transferred electrophoretically on nitrocellulose membranes (Schleicher \& Schuell BioScience GmbH, Germany). Membranes were blocked with 5\% BSA (Sigma-Aldrich) in TBS-1X Tween-20 $0.1 \%$ and incubated overnight at $4^{\circ} \mathrm{C}$ with guinea pig nephrin pAb (1:500) (Progen Biotechnik GmbH, Germany), rabbit podocin pAb (1:500) (Abcam, Cambridge, UK), and with mouse anti-actin mAb (1:3000) (C4; Chemicon International, Temecula, CA). Appropriate HRPlinked antibodies (Cell Signaling Technology) were applied for 60 minutes at room temperature. Signal was detected using appropriate chemiluminescence reagent (Amersham Biosciences, GE Healthcare, UK). Bands were normalized to actin expression. Image-J (NIH, MD, USA) densitometry analysis system was used for measurements.

2.4. Real Time RT-PCR ( $q R T-P C R)$. Renal tissue was homogenized in Trizol Reagent (Life Technologies; Gibco BRL, Paisley, UK). One $\mu \mathrm{g}$ of total RNA was reverse transcribed (Superscript-II; Gibco) and amplified by RT-PCR. Products were normalized according to glyceraldehyde-3-phosphate dehydrogenase (GAPDH) expression. Measurements were performed using the ABI-Prism 7000 System (Applied Biosystems; California, USA). iTaq SYBR-Green Supermix with ROX (Bio-Rad) was used for the reactions. Results were normalized to GAPDH and analysis was performed using the $2^{-\Delta \Delta \mathrm{Ct}}$ method. All samples were tested in duplicate. 
TABLE 1: Clinical and biochemical characteristics of groups under study.

\begin{tabular}{lcccccc}
\hline & \multicolumn{3}{c}{ Group } & & \multicolumn{2}{c}{$P$} \\
& PHN & PHN-Rapa & HC & \multicolumn{2}{c}{ PHN versus } & PHN versus \\
& & & & PHN-Rapa & HC & versus HC \\
\hline Initial body weight (gr) & $170 \pm 2.8$ & $169 \pm 5.2$ & $180 \pm 2.1$ & 0.9 & 0.12 & 0.1 \\
Final body weight (gr) & $335.7 \pm 7.3$ & $268.3 \pm 12.1$ & $432.5 \pm 9.2$ & $<0.001$ & $<0.001$ & $<0.001$ \\
Kidney mass (gr) & $4.3 \pm 0.3$ & $2.8 \pm 0.2$ & $4.04 \pm 0.02$ & 0.001 & 0.47 & 0.009 \\
Kidney mass over body weight & $0.013 \pm 0.0002$ & $0.010 \pm 0.0004$ & $0.009 \pm 0.0003$ & 0.013 & 0.002 & 0.27 \\
Serum creatinine (mg/dL) & $0.30 \pm 0.001$ & $0.31 \pm 0.03$ & $0.40 \pm 0.001$ & 0.55 & 0.006 & 0.016 \\
Serum total protein (mg/dL) & $5.91 \pm 0.14$ & $6.33 \pm 0.12$ & $6.77 \pm 0.075$ & 0.032 & 0.001 & 0.04 \\
Serum albumin (mg/dL) & $2.8 \pm 0.09$ & $3.1 \pm 0.1$ & $3.6 \pm 0.04$ & 0.03 & 0.002 & 0.04 \\
Serum cholesterol (mg/dL) & $187.5 \pm 21$ & $313.3 \pm 53$ & $66 \pm 5$ & 0.03 & 0.05 & 0.001 \\
\hline
\end{tabular}

2.5. Microscopy Studies. Left kidney sections were fixed in neutral formalin and examined by a renal pathologist (L. Nakopoulou) who was blinded to the group assignment.

For immunofluorescence (IF) studies left kidney sections were embedded in OCT compound (Sakura Finetek USA, Inc), snap frozen in liquid nitrogen, and stored in $-80^{\circ} \mathrm{C}$ until examination. Five-micrometer thick cryosections were incubated overnight with the same primary antibodies used in western blot. Dilution for nephrin was 1:250 and for podocin was $1: 100$. Secondary antibodies used included Alexa Fluor- 488 conjugated goat anti-rabbit or anti-guinea pig or anti-rat IgG at 1/1000 (Molecular Probes, Inc). RNAse (Sigma) diluted in BSA1\% PBS Tween1X (1:500) was applied for $30 \mathrm{~min}$ and then samples were incubated with propidium iodide 1:1000 (Sigma) for 5 minutes. At least 30 glomeruli were examined per animal. The intensity of the fluorescence was scored on a scale of 0 to $3+$, where $0=$ absent, $1+=$ mild, $2+=$ moderate, and $3+=$ strong staining.

For EM studies left kidney sections were processed as usual and examined under a transmission electron microscope (JEM100CX-II; JEOL Inc., Tokyo, Japan). Twenty random glomeruli were examined for each mouse. Microphotographs were analyzed using the Digital Micrograph software (Gatan $\mathrm{GmbH}$, Munchen, Germany). The entire curved length of the GBM of all open capillary loops (loop length, LL) and the number of foot processes (FPN) overlying capillary loops were measured. The foot process width (FPW) in each loop was calculated using the formula: $\mathrm{FPW}=(\pi / 4 \times$ LL)/FPN [23]. The foot process density (FPD) in each loop was measured using the formula: FPD $=$ FPN/LL.

2.6. Measurement of Proteinuria, Serum Creatinine, and Rapamycin Levels. Urinary protein concentration was determined with the Bio-Rad protein assay (Bio-Rad, Hercules, CA). Serum creatinine levels were measured at sacrifice by an autoanalyzer (Olympus 600, Tokyo, Japan). Rapamycin blood levels were determined by EIA in whole blood (Imx Analyzer, Abbott Lab, USA).

2.7. Statistical Analysis. Analysis of variance (ANOVA) was performed to compare serum creatinine and IF scores between groups. Continuous variables are expressed as
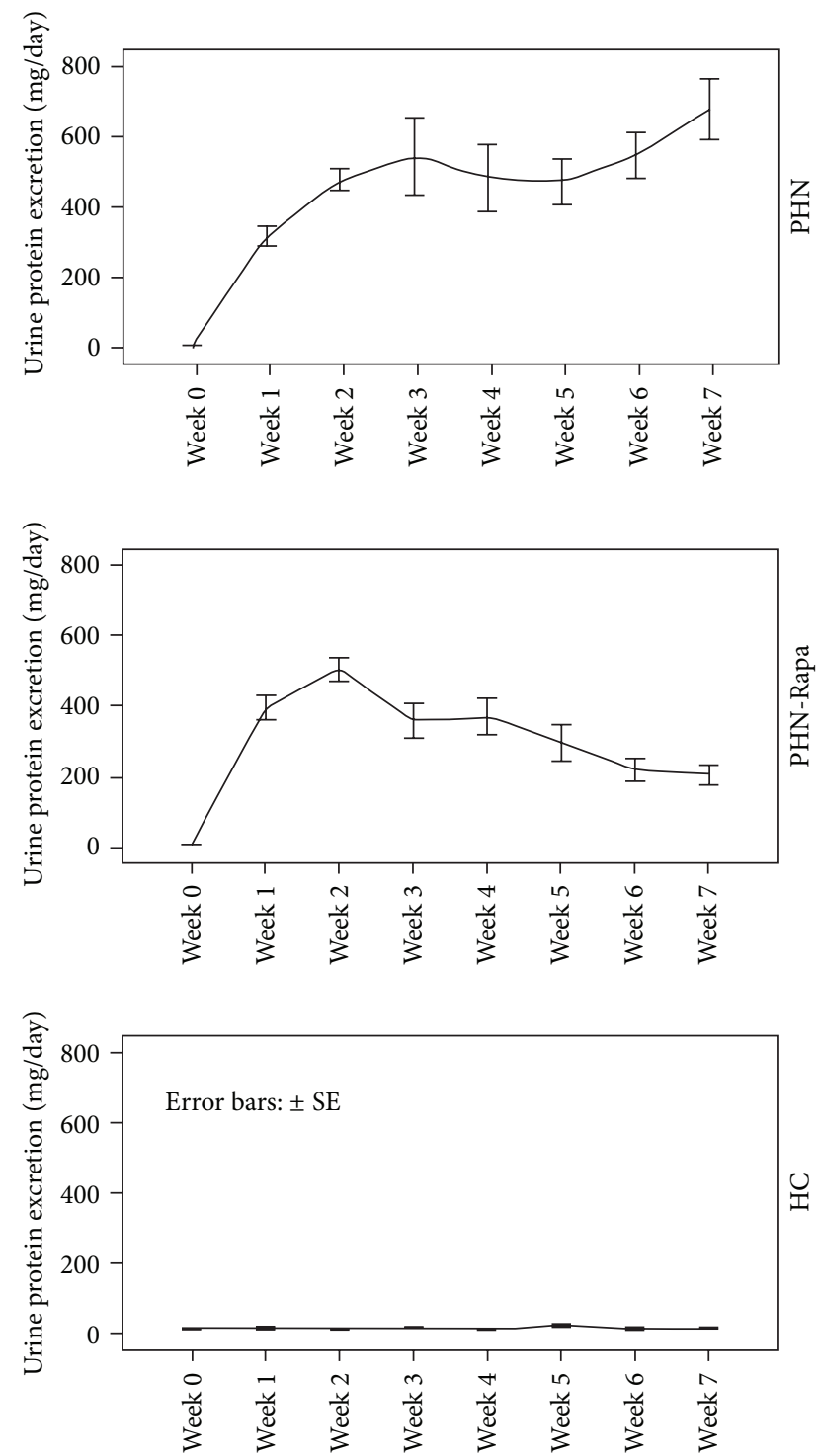

FIGURE 1: 24-hour urine protein excretion in study groups. Rapamycin was administered at week one in the PHN-Rapa group resulting in gradual amelioration of proteinuria in contrast to the PHN group in which proteinuria continued deteriorating. 


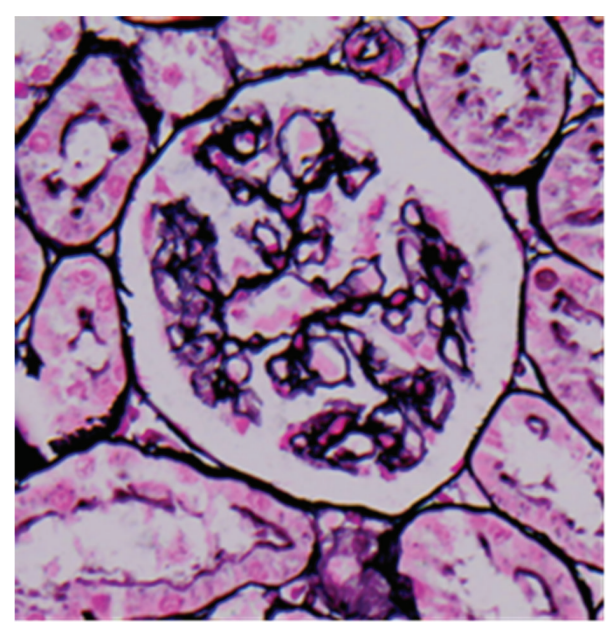

(a)

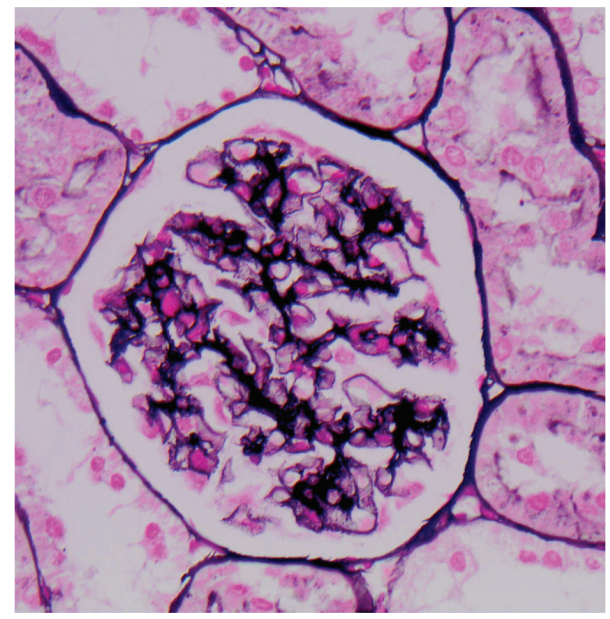

(b)

Figure 2: Photon microscopy. (a) Passive Heymann Nephritis. Moderate to severe irregular thickening of glomerular capillary basement membranes (Silver Methenamine, $\times 400$ ). (b) Passive Heymann Nephritis after rapamycin administration. Mild to moderate thickening of glomerular capillary basement membranes (Silver Methenamine, $\times 400$ ).

mean \pm SE. Repeated measures analysis of variance was used to compare the weekly measurements of proteinuria and body weight throughout the study. Independent samples Kruskal-Wallis and median tests were used for nonparametric comparisons. Differences were considered significant for a $P$ less than 0.05 (two tailed). SPSS19-IBM software was used for statistical analysis.

\section{Results}

3.1. Clinical and Biochemical Characteristics. The 24-hour urinary protein of both PHN and PHN-Rapa groups increased at nephrotic levels at day 7 and remained so until the 2 nd week after nephritis induction. Thereafter proteinuria began to decline in the PHN-Rapa group while it continued deteriorating in the PHN group. At the end of the study (week 7) urine protein levels in the PHN-Rapa group were 1/3 of those in the PHN group $(P=0.007$, by repeated measures ANOVA). Although proteinuria in the PHN-Rapa group declined continuously, it did not reach the urine protein levels of HCs at the time of sacrifice (Figure 1).

Rats in the PHN-Rapa group did not increase BW at the same pace as the other groups. At the end of the study their BW was $66 \%$ and $45 \%$ of that in the PHN and $\mathrm{HC}$ groups (Table 1). The ratio of renal to body mass at the end of the study was higher $(P<0.01)$ in the PHN group (0.013) compared to PHN-Rapa group (0.010) and HC group (0.009). Serum creatinine, total protein, and total cholesterol are presented in Table 1. Serum creatinine levels were higher in the $\mathrm{HC}$ group due to the higher body weight in this group at the end of the study. Serum total protein and albumin levels in the PHN-Rapa group were significantly higher as compared to the PHN group but did not reach those of HCs (Table 1). The trough rapamycin levels averaged at $12.5 \pm 0.76 \mathrm{ng} / \mathrm{mL}$ in treated rats.

\subsection{Photon Microscopy, Immunofluorescence, and Electron} Microscopy. After staining with silver methenamine the glomeruli in the PHN group revealed moderate to severe thickening of the GBM, while rapamycin treated rats displayed less severe histological lesions with only mild or moderate thickening (Figure 2). Cryosections stained for anti-Rat IgG showed intense (3+) granular and irregular fluorescence along the glomerular capillary walls of the PHN group, whereas staining was attenuated $(1+$ to $2+)$ in the PHN-Rapa group and absent in the control group (Figure 3 ). Electron microscopy showed massive subepithelial deposits in almost all capillary loops in the PHN group with severely affected podocytes (Figure 4). In particular the harmonic mean and the median value of FPW were $830.78 \mathrm{~nm}$ and $789.26 \mathrm{~nm}$, respectively, while the mean foot process density per $\mu \mathrm{m}$ of GBM length (FPD) was $0.94 \pm 0.42$. In the PHNRapa group the deposits and the podocytic injury were markedly attenuated compared to the PHN group (harmonic mean of FPW $613.3 \mathrm{~nm}$, median $654.1 \mathrm{~nm}$, and FPD 1.28 \pm 0.9 ; all $P<0.001)$. Despite this improvement, the respective values in the HC were much lower (harmonic mean FPW $349 \mathrm{~nm}$, median FPW $355 \mathrm{~nm}$, and mean FPD $2.24 \pm 0.43$; all $P<0.001$ as compared to other groups).

3.3. Nephrin and Podocin Expression. By WB the levels of nephrin and podocin protein levels in glomerular lysates were significantly lower (all $P<0.05$ ) in the PHN group as compared to the $\mathrm{HC}$ and $\mathrm{PHN}-\mathrm{Rapa}$ groups. In the PHNRapa group, nephrin and podocin levels were similar to HCs (Figure 5).

By RT-PCR the expression of nephrin mRNA was lower in the PHN group compared to $\mathrm{HC}$ and PHN-Rapa groups $(P=0.011$ and $P=0.039$, resp.; Figure 6). In contrast podocin mRNA was increased in the PHN and PHN-RAPA groups as compared to HCs (Figure 6).

Immunofluorescence for nephrin and podocin showed intense $(3+)$ and regular linear staining in all examined glomeruli in the HC group. Normal staining for both proteins was also evident in the majority of glomeruli $(72.8 \%)$ in the 


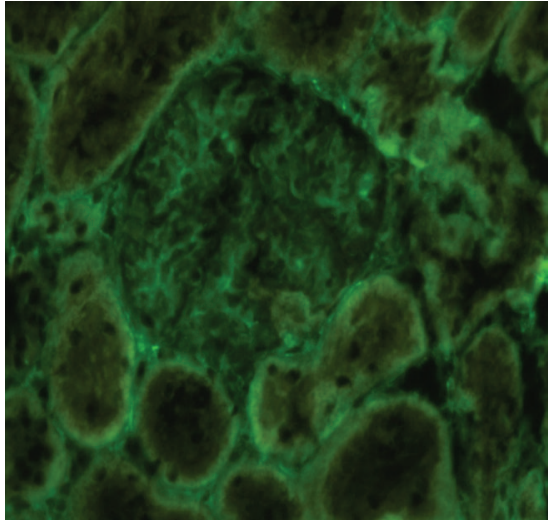

(a)

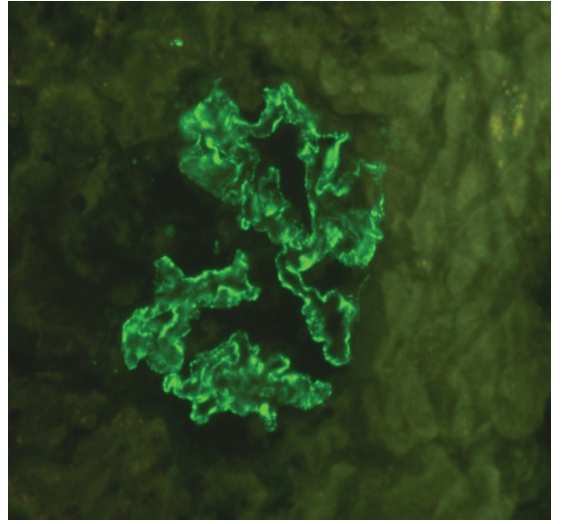

(b)

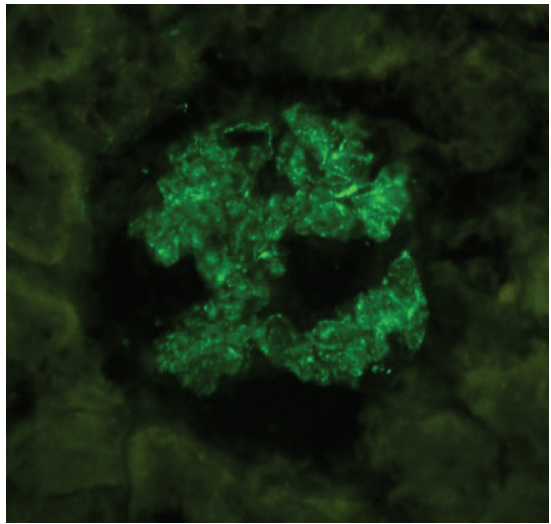

(c)

FIGURE 3: Immunofluorescence staining with anti-Rat IgG. (a) Healthy controls showed absence of staining. Original magnification $\times 400$. (b) Passive Heymann Nephritis; glomeruli showed intense (3+) granular and irregular fluorescence along the capillary walls $(\times 400)$. (c) Passive Heymann Nephritis after rapamycin administration; staining was significantly attenuated (1+ to $2+)$ in almost all glomeruli $(\times 400)$.

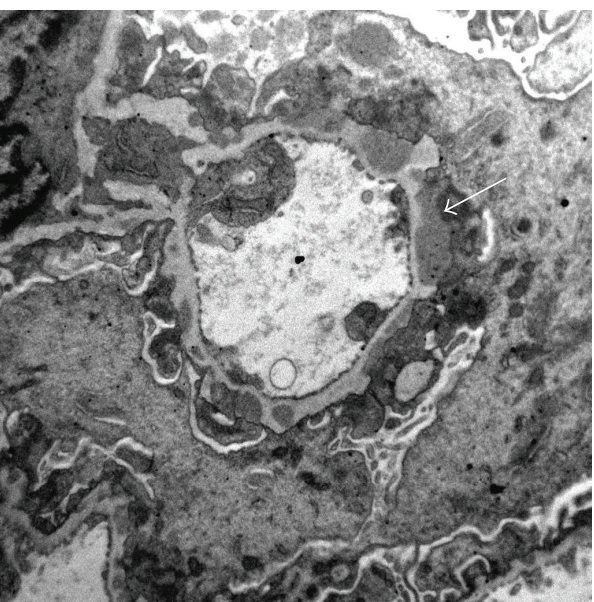

(a)

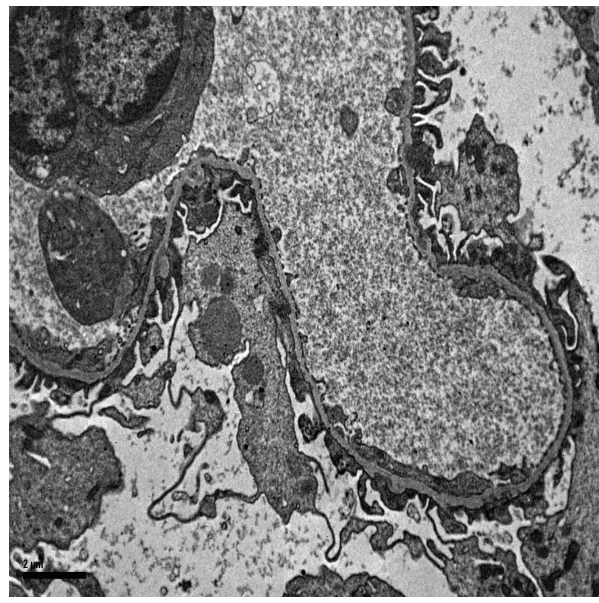

(c)

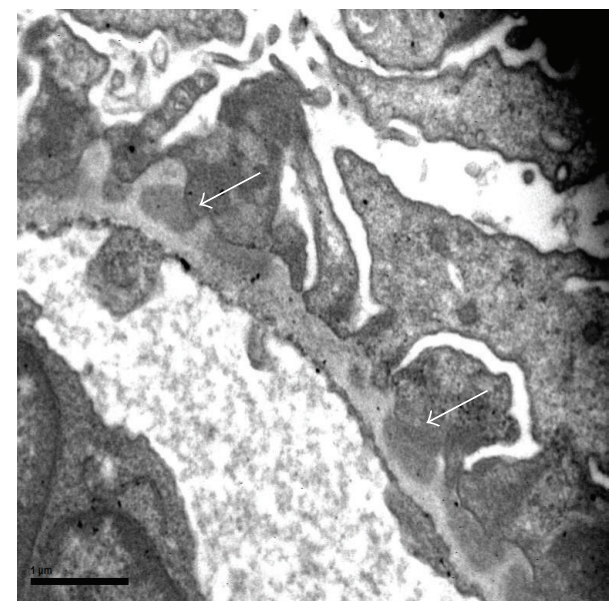

(b)

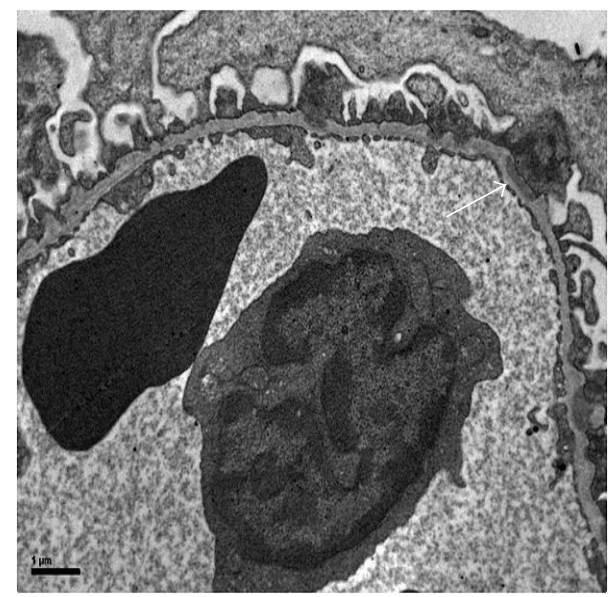

(d)

Figure 4: Electron microscopy. (a) Passive Heymann Nephritis. Massive subepithelial deposits (white arrow) with severe fusion of podocyte foot processes; original magnification $\times 12 \mathrm{k}$. (b) Passive Heymann Nephritis. Subepithelial deposits in higher magnification (white arrows); original magnification $\times 26 \mathrm{k}$. (c) Passive Heymann Nephritis after rapamycin administration. The deposits and the podocytic injury were attenuated compared to the PHN group; original magnification $\times 8 \mathrm{k}$. (d) Passive Heymann Nephritis after rapamycin administration (higher magnification $\times 20 \mathrm{k}$ ). Small subepithelial deposit (white arrow). 

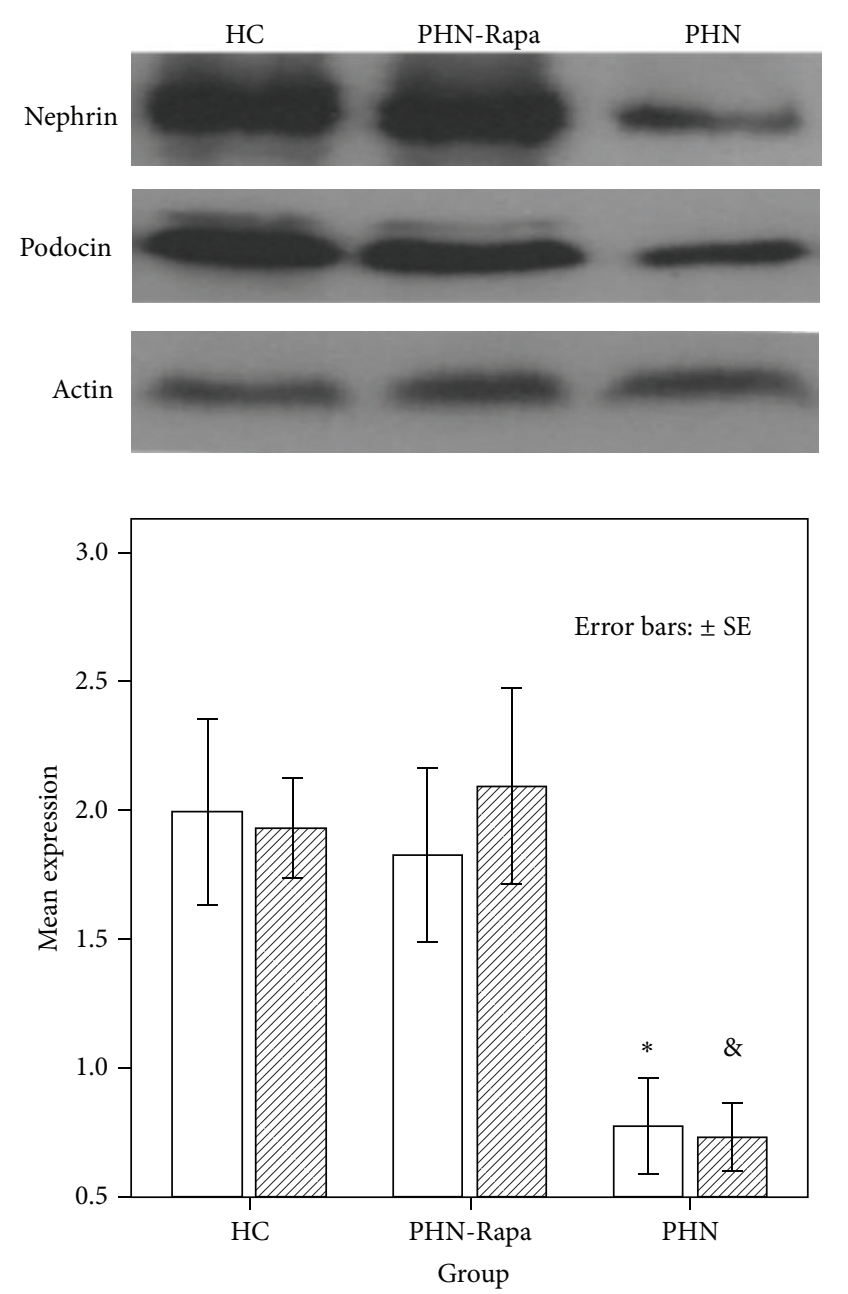

$\square$ Nephrin
$\square$ Podocin

FIGURE 5: Western blot analysis for nephrin, podocin, and actin in glomerular lysates. HC: healthy controls; PHN: Passive Heymann Nephritis; PHN-Rapa: Passive Heymann Nephritis after rapamycin administration. Bars represent nephrin (white columns) and podocin (shaded columns) protein content corrected to actin concentration. ${ }^{*} P=0.01,{ }^{\&} P=0.007$. Error bars: \pm SE.

PHN-Rapa group. On the contrary, staining for nephrin and podocin was irregular and attenuated $(<3+)$ in almost all glomeruli in the PHN group (Figure 7).

\section{Discussion}

Rapamycin treatment has shown either protective $[21,22,24-$ 31] or untoward [32-36] results in various forms of experimental or human kidney disease. In summary, rapamycin displays dual opposing effects, with proteinuria and podocyte damage aggravation in the toxicoimmunological glomerular models and a nephroprotective effect in the chronic inflammatory glomerulotubulointerstitial models [37]. Rapamycin inhibits the proliferation of both $\mathrm{T}$ and $\mathrm{B}$ cells [19] and reduces the number of $\mathrm{B}$ cells capable of producing immunoglobulins in contrast to cyclosporine and tacrolimus [20].

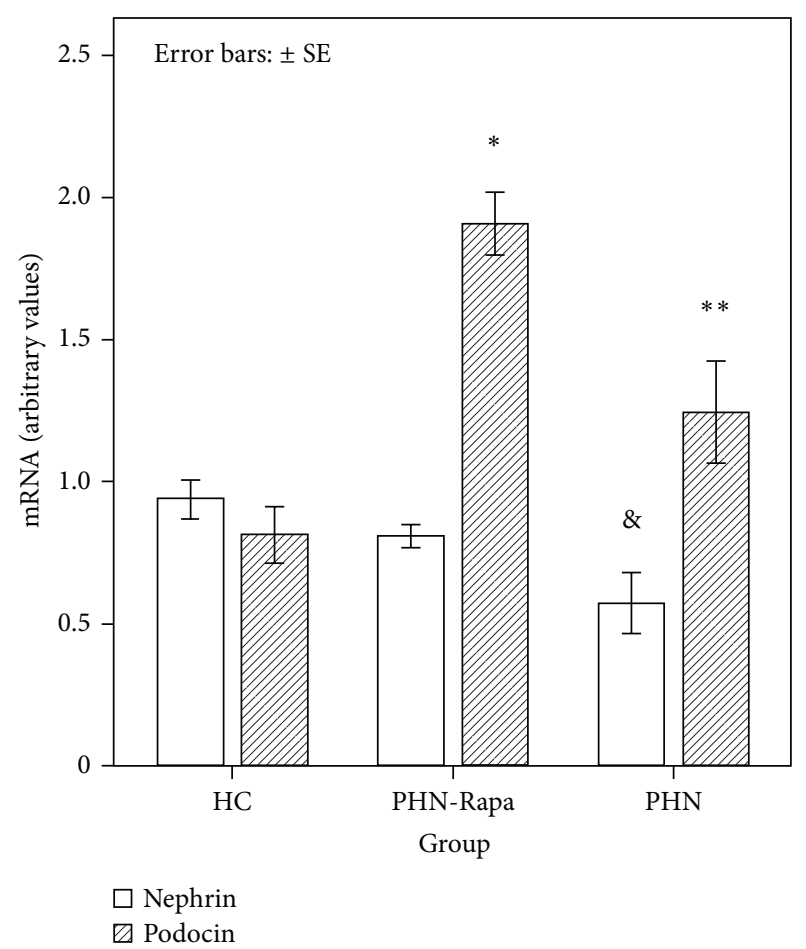

FIgURE 6: Real time PCR for nephrin and podocin mRNA in glomerular lysates. HC: healthy controls; PHN: Passive Heymann Nephritis; PHN-Rapa: Passive Heymann Nephritis after rapamycin administration. Bars represent nephrin mRNA (white columns) and podocin mRNA (shaded columns) corrected to GAPDH mRNA concentration. ${ }^{*} P<0.001,{ }^{\&} P=0.011,{ }^{* *} P=0.038$. Error bars: \pm SE.

Rapamycin can also promote the generation of regulatory $\mathrm{T}$ cells which suppress the immune system and control autoimmunity [38]. These combined properties make sirolimus an attractive agent for the treatment of autoimmune diseases such as MN.

In the present study rapamycin was given after induction of $\mathrm{HN}$ when severe proteinuria, and by inference histological lesions, had already been established. Nevertheless, rapamycin was able to abrogate the second rise of proteinuria during the autologous phase. This clinical result was escorted by significant alleviation of the histological lesions. More precise podocyte indices such as FPW and FPD were markedly improved by rapamycin whereas the expression of slit diaphragm proteins nephrin and podocin was almost completely restored. It is interesting that in the PHN group, podocin mRNA levels were increased, nephrin mRNA levels were decreased, and the respective protein levels were both decreased. This discrepancy implies that nephrin decreased owing to suppressed translation, whereas the decreased podocin levels may be due to loss or destruction of the protein with a compensatory increase in podocin mRNA levels. Residual histological lesions seen in the PHN-Rapa group should be attributed to the initial insult during the heterologous phase when the drug had not been given yet. The attenuation of anti-Rat IgG staining in IF indicates that rapamycin blocked the production of pathogenic autologous 

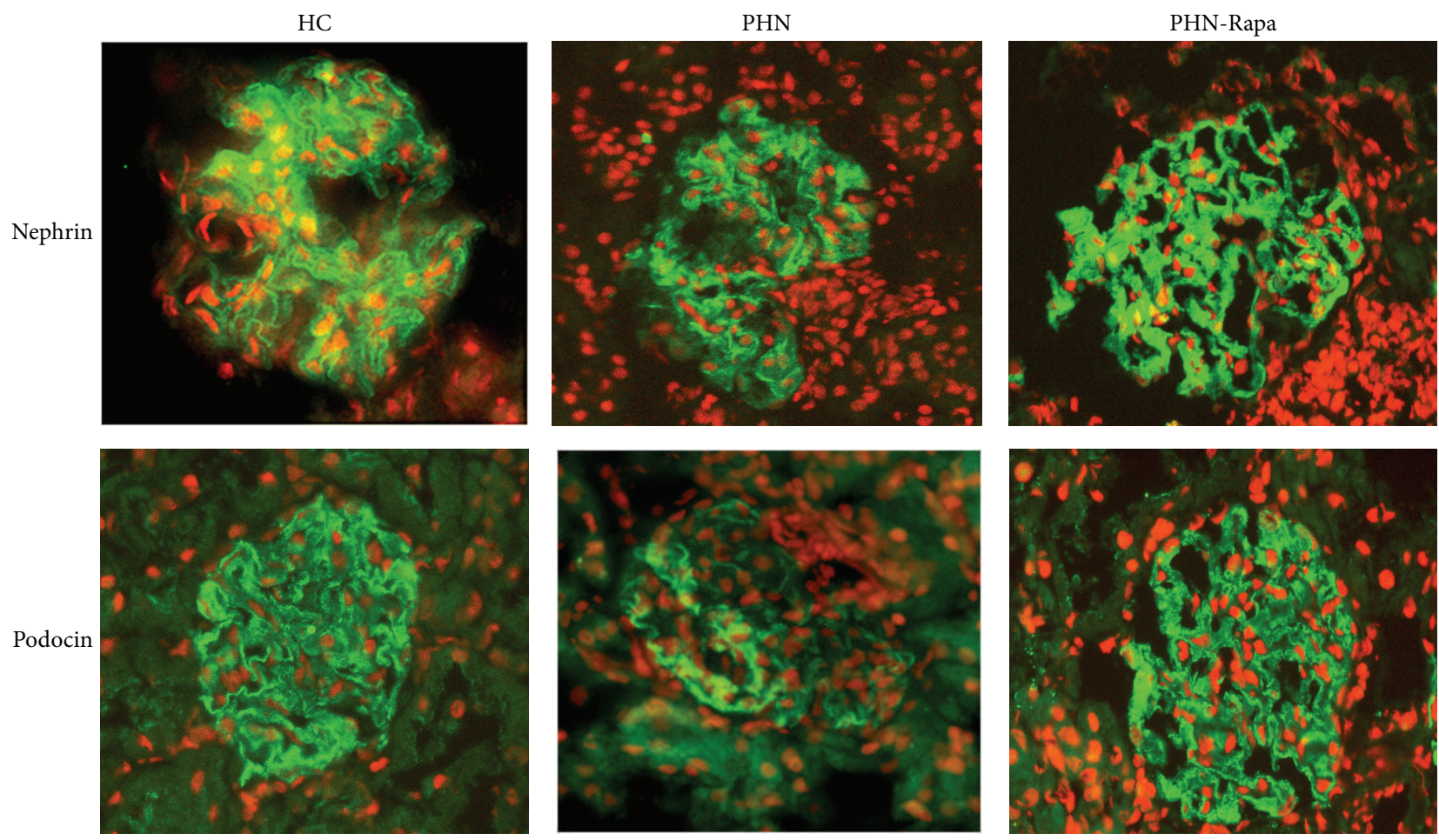

FIGURE 7: Immunofluorescence staining for nephrin and podocin. HC: healthy controls; PHN: Passive Heymann Nephritis; PHN-Rapa: Passive Heymann Nephritis after rapamycin administration. Nuclei have been stained with propidium iodide (red). Intense (3+) linear staining in the HC and PHN-Rapa groups in contrast to the PHN group which presented attenuated and irregular pattern of staining for both nephrin and podocin (middle panel).

alloantibodies (possibly via its B-cell inhibitory effects) that are responsible for the second boost of proteinuria, resulting thus in the gradual resolution of the NS. These results are in line with previous studies in animal models of MN [21, 22] and offer further insights into possible mechanisms for the therapeutic effect of rapamycin in experimental MN.

\section{Conclusions}

Rapamycin significantly improves proteinuria and histological lesions during the autologous phase of $\mathrm{PHN}$, an effect that may be mediated by inhibition of the autoimmune response and by restoration of the normal expression of the podocyte proteins nephrin and podocin. If our results are confirmed by future studies, rapamycin may prove to be an effective treatment for MN.

\section{Conflict of Interests}

The authors declare that there is no conflict of interests.

\section{Acknowledgment}

This work was supported by the Secretariat of the Research Committee (Code 2976) of the University of Crete in Greece.

\section{References}

[1] C. Ponticelli, "Membranous nephropathy," Journal of Nephrology, vol. 20, no. 3, pp. 268-287, 2007.
[2] A. Schieppati, L. Mosconi, A. Perna et al., "Prognosis of untreated patients with idiopathic membranous nephropathy," The New England Journal of Medicine, vol. 329, no. 2, pp. 85-89, 1993.

[3] C. Ponticelli, P. Zucchelli, P. Passerini et al., "A 10-year follow-up of a randomized study with methylprednisolone and chlorambucil in membranous nephropathy," Kidney International, vol. 48, no. 5, pp. 1600-1604, 1995.

[4] V. Jha, A. Ganguli, T. K. Saha et al., "A randomized, controlled trial of steroids and cyclophosphamide in adults with nephrotic syndrome caused by idiopathic membranous nephropathy," Journal of the American Society of Nephrology, vol. 18, no. 6, pp. 1899-1904, 2007.

[5] N. Polanco, E. Gutiérrez, A. Covarsí, F. Ariza, A. Carreño, A. Vigil et al., "Spontaneous remission of nephrotic syndrome in idiopathic membranous nephropathy," Journal of the American Society of Nephrology, vol. 21, no. 4, pp. 697-704, 2010.

[6] S. L. Hogan, K. E. Muller, J. C. Jennette, and R. J. Falk, "A review of therapeutic studies of idiopathic membranous glomerulopathy," American Journal of Kidney Diseases, vol. 25, no. 6, pp. 862875,1995

[7] D. C. Cattran, Y. Pei, C. M. T. Greenwood, C. Ponticelli, P. Passerini, and E. Honkanen, "Validation of a predictive model of idiopathic membranous nephropathy: its clinical and research implications," Kidney International, vol. 51, no. 3, pp. 901-907, 1997.

[8] H. Shiiki, T. Saito, Y. Nishitani et al., "Prognosis and risk factors for idiopathic membranous nephropathy with nephrotic syndrome in Japan," Kidney International, vol. 65, no. 4, pp. 14001407, 2004. 
[9] L. H. Beck Jr., R. G. B. Bonegio, G. Lambeau et al., "M-type phospholipase A2 receptor as target antigen in idiopathic membranous nephropathy," The New England Journal of Medicine, vol. 361, no. 1, pp. 11-21, 2009.

[10] H. Debiec, J. Nauta, F. Coulet et al., "Role of truncating mutations in MME gene in fetomaternal alloimmunisation and antenatal glomerulopathies," The Lancet, vol. 364, no. 9441, pp. 1252-1259, 2004.

[11] H. Debiec, F. Lefeu, M. J. Kemper et al., "Early-childhood membranous nephropathy due to cationic bovine serum albumin," The New England Journal of Medicine, vol. 364, no. 22, pp. 21012110, 2011.

[12] M. Prunotto, M. L. Carnevali, G. Candiano et al., "Autoimmunity in membranous nephropathy targets aldose reductase and SOD2," Journal of the American Society of Nephrology, vol. 21, no. 3, pp. 507-519, 2010.

[13] P. Ronco and H. Debiec, "Antigen identification in membranous nephropathy moves toward targeted monitoring and new therapy," Journal of the American Society of Nephrology, vol. 21, no. 4, pp. 564-569, 2010.

[14] P. Ronco and H. Debiec, "Pathogenesis of membranous nephropathy: recent advances and future challenges," Nature Reviews Nephrology, vol. 8, no. 4, pp. 203-213, 2012.

[15] W. Heymann, D. B. Hackel, S. Harwood, S. G. Wilson, and J. L. Hunter, "Production of nephrotic syndrome in rats by Freund's adjuvants and rat kidney suspensions," Proceedings of the Society for Experimental Biology and Medicine, vol. 100, no. 4, pp. 660$664,1959$.

[16] D. J. Salant, R. J. Quigg, and A. V. Cybulsky, "Heymann nephritis: mechanisms of renal injury," Kidney International, vol. 35, no. 4, pp. 976-984, 1989.

[17] P. Ruggenenti, P. Cravedi, A. Chianca, A. Perna, B. Ruggiero, F. Gaspari et al., "Rituximab in idiopathic membranous nephropathy," Journal of the American Society of Nephrology, vol. 23, no. 8, pp. 1416-1425, 2012.

[18] L. H. Beck Jr., F. C. Fervenza, D. M. Beck et al., "Rituximabinduced depletion of anti-PLA2R autoantibodies predicts response in membranous nephropathy," Journal of the American Society of Nephrology, vol. 22, no. 8, pp. 1543-1550, 2011.

[19] K. M. Aagaard-Tillery and D. F. Jelinek, "Inhibition of human B lymphocyte cell cycle progression and differentiation by rapamycin," Cellular Immunology, vol. 156, no. 2, pp. 493-507, 1994.

[20] S. Heidt, D. L. Roelen, C. Eijsink, C. Van Kooten, F. H. J. Claas, and A. Mulder, "Effects of immunosuppressive drugs on purified human b cells: evidence supporting the use of $\mathrm{mmf}$ and rapamycin," Transplantation, vol. 86, no. 9, pp. 1292-1300, 2008.

[21] R. G. B. Bonegio, R. Fuhro, Z. Wang et al., "Rapamycin ameliorates proteinuria-associated tubulointerstitial inflammation and fibrosis in experimental membranous nephropathy," Journal of the American Society of Nephrology, vol. 16, no. 7, pp. 20632072, 2005.

[22] R. Naumovic, D. Jovovic, G. Basta-Jovanovic et al., "Effects of rapamycin on active Heymann nephritis," American Journal of Nephrology, vol. 27, no. 4, pp. 379-389, 2007.

[23] J. K. J. Deegens, H. B. P. M. Dijkman, G. F. Borm et al., "Podocyte foot process effacement as a diagnostic tool in focal segmental glomerulosclerosis," Kidney International, vol. 74, no. 12, pp. 1568-1576, 2008.

[24] J. Rovira, E. M. Arellano, J. Carreras et al., "Mammalian target of rapamycin inhibition prevents glomerular hypertrophy in a model of renal mass reduction," Transplantation, vol. 88, no. 5, pp. 646-652, 2009.

[25] M.-J. Wu, M.-C. Wen, Y.-T. Chiu, Y.-Y. Chiou, K.-H. Shu, and M.-J. Tang, "Rapamycin attenuates unilateral ureteral obstruction-induced renal fibrosis," Kidney International, vol. 69, no. 11, pp. 2029-2036, 2006.

[26] N. Lloberas, J. M. Cruzado, M. Franquesa et al., "Mammalian target of rapamycin pathway blockade slows progression of diabetic kidney disease in rats," Journal of the American Society of Nephrology, vol. 17, no. 5, pp. 1395-1404, 2006.

[27] S. L. Lui, S. Yung, R. Tsang et al., "Rapamycin prevents the development of nephritis in lupus-prone NZB/W F 1 mice," Lupus, vol. 17, no. 4, pp. 305-313, 2008.

[28] S. L. Lui, R. Tsang, K. W. Chan et al., "Rapamycin attenuates the severity of established nephritis in lupus-prone NZB/W F1 mice," Nephrology Dialysis Transplantation, vol. 23, no. 9, pp. 2768-2776, 2008.

[29] K. Stylianou, I. Petrakis, V. Mavroeidi et al., “The PI3K/Akt/ mTOR pathway is activated in murine lupus nephritis and downregulated by rapamycin," Nephrology Dialysis Transplantation, vol. 26, no. 2, pp. 498-508, 2011.

[30] S. Krämer, Y. Wang-Rosenke, V. Scholl et al., "Low-dose mTOR inhibition by rapamycin attenuates progression in anti-thylinduced chronic glomerulosclerosis of the rat," American Journal of Physiology, vol. 294, no. 2, pp. F440-F449, 2008.

[31] S. Wittmann, C. Daniel, A. Braun et al., "The mTOR inhibitor everolimus attenuates the time course of chronic anti-Thyl nephritis in the rat," Nephron, vol. 108, no. 2, pp. e45-e56, 2008.

[32] R. Vogelbacher, S. Wittmann, A. Braun, C. Daniel, and C. Hugo, "The mTOR inhibitor everolimus induces proteinuria and renal deterioration in the remnant kidney model in the rat," Transplantation, vol. 84, no. 11, pp. 1492-1499, 2007.

[33] C. Daniel, R. Ziswiler, B. Frey, M. Pfister, and H.-P. Marti, "Proinflammatory effects in experimental mesangial proliferative glomerulonephritis of the immunosuppressive agent SDZ RAD, a rapamycin derivative," Experimental Nephrology, vol. 8, no. 1, pp. 52-62, 2000.

[34] C. Daniel, L. Renders, K. Amann, E. Schulze-Lohoff, I. A. Hauser, and C. Hugo, "Mechanisms of everolimus-induced glomerulosclerosis after glomerular injury in the rat," American Journal of Transplantation, vol. 5, no. 12, pp. 2849-2861, 2005.

[35] F. C. Fervenza, P. M. Fitzpatrick, J. Mertz et al., "Acute rapamycin nephrotoxicity in native kidneys of patients with chronic glomerulopathies," Nephrology Dialysis Transplantation, vol. 19, no. 5, pp. 1288-1292, 2004.

[36] K. Stylianou, I. Petrakis, V. Mavroeidi, S. Stratakis, G. Kokologiannakis, E. Lioudaki et al., "Rapamycin induced ultrastructural and molecular alterations in glomerular podocytes in healthy mice," Nephrology Dialysis Transplantation, vol. 27, no. 8, pp. 3141-3148, 2012.

[37] J. Torras, I. Herrero-Fresneda, O. Gulias et al., "Rapamycin has dual opposing effects on proteinuric experimental nephropathies: is it a matter of podocyte damage," Nephrology Dialysis Transplantation, vol. 24, no. 12, pp. 3632-3640, 2009.

[38] M. Battaglia, A. Stabilini, B. Migliavacca, J. Horejs-Hoeck, T. Kaupper, and M.-G. Roncarolo, "Rapamycin promotes expansion of functional CD4+CD25 +FOXP3+ regulatory T cells of both healthy subjects and type 1 diabetic patients," Journal of Immunology, vol. 177, no. 12, pp. 8338-8347, 2006. 


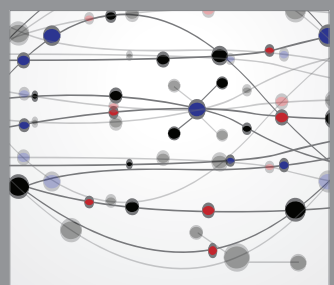

The Scientific World Journal
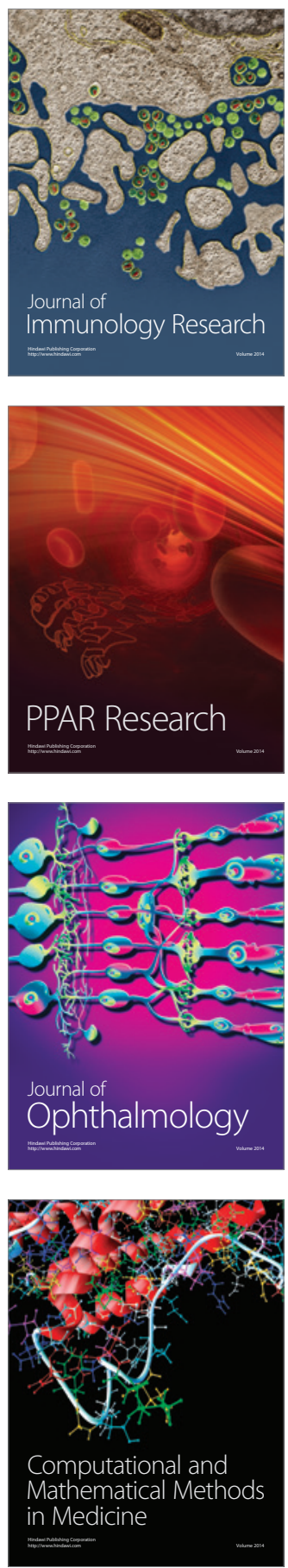

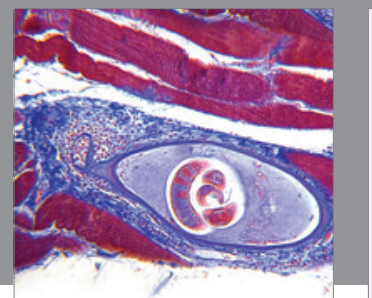

Gastroenterology

Research and Practice
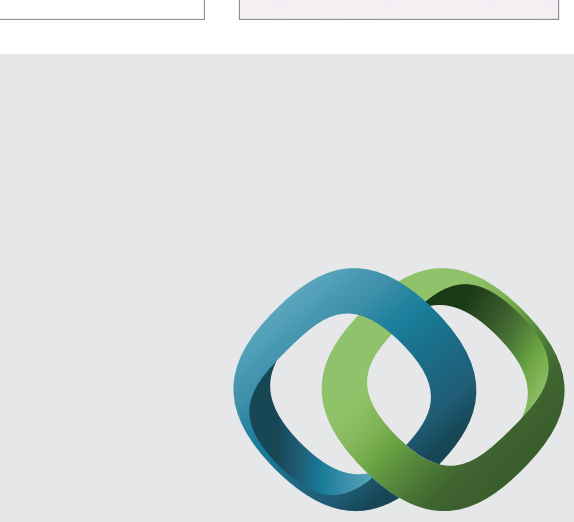

\section{Hindawi}

Submit your manuscripts at

http://www.hindawi.com
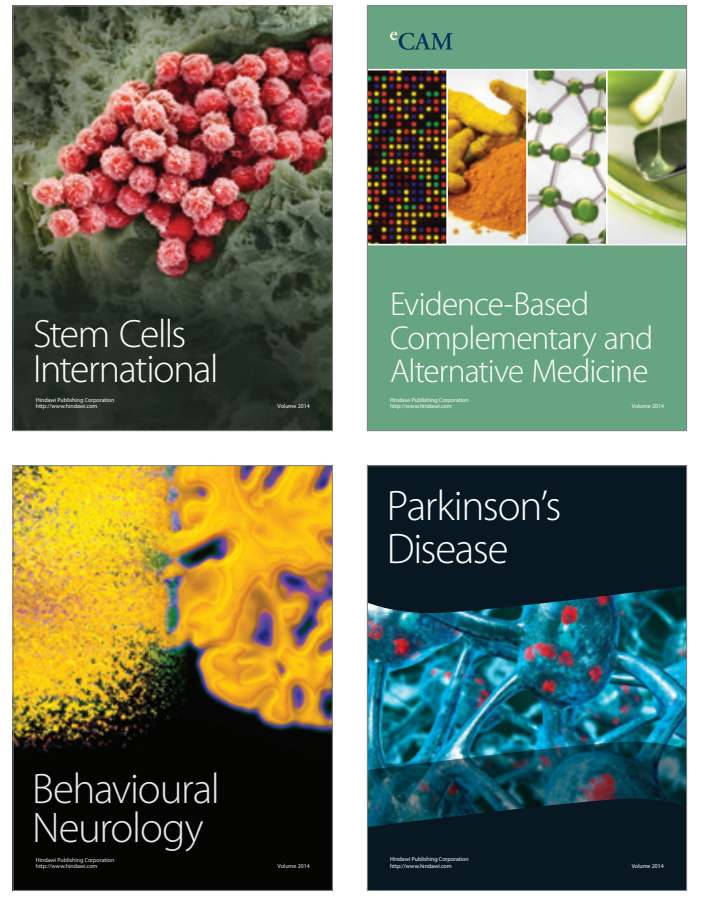
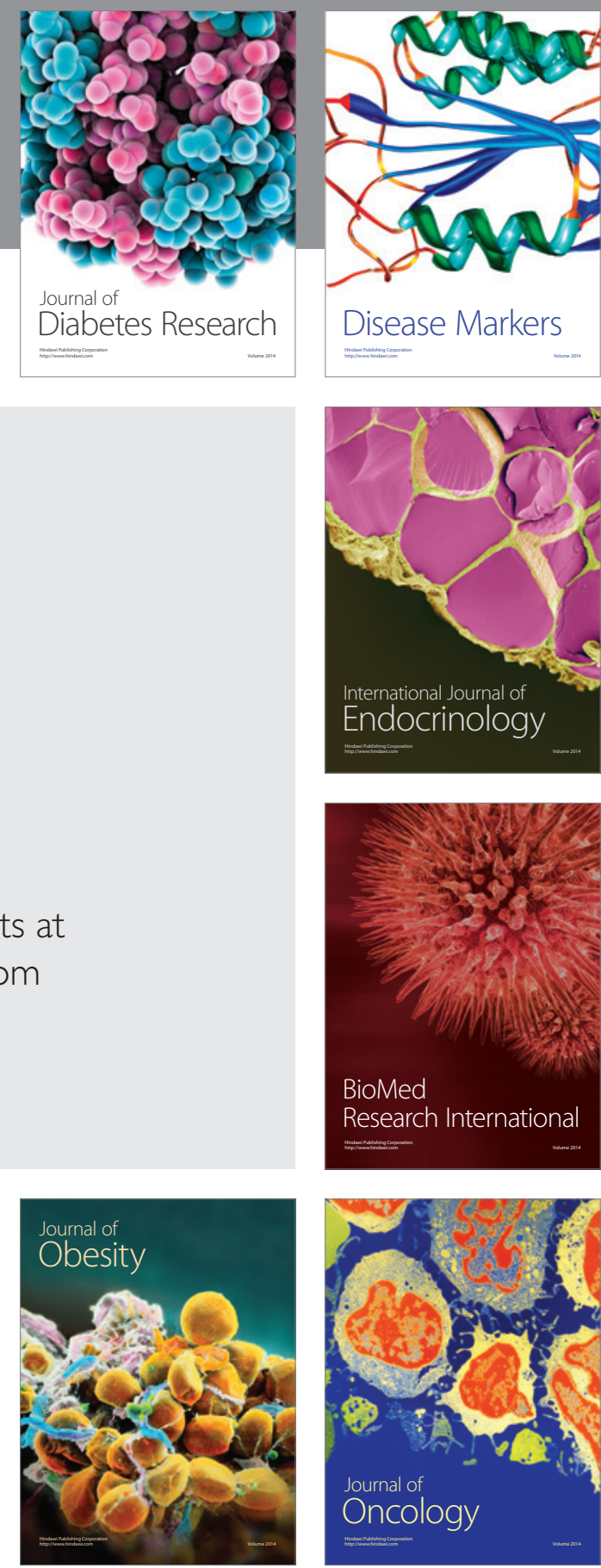

Disease Markers
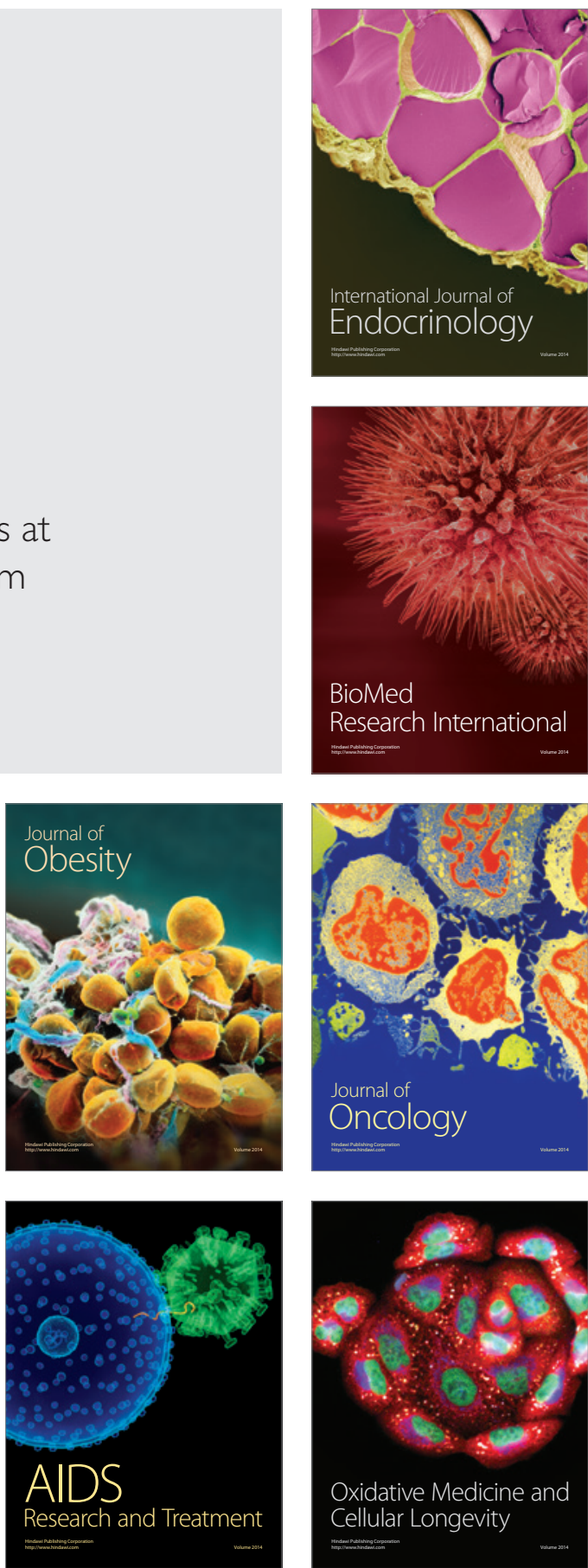